ORIGINAL

\title{
Recurrent pregnancy-induced diabetes insipidus in a woman with hemochromatosis
}

\author{
Robert Krysiak, Iwona Kobielusz-Gembala and Boguslaw Okopien \\ Department of Internal Medicine and Clinical Pharmacology, Medical University of Silesia, Katowice, Poland
}

\begin{abstract}
Diabetes insipidus is a rare disorder in pregnant women, predating pregnancy or appearing for the first time during gestation. In pregnancy it usually affects women with HELLP syndrome or acute fatty liver of pregnancy and results from the reduced hepatic degradation of placental vasopressinase leading to its increased activity. Although infiltrative diseases have been found to cause diabetes insipidus in non-pregnant population, very few studies showed that these disorders may manifest for the first time during gestation. We describe here the case of transient diabetes insipidus in two subsequent pregnancies of a female with hemochromatosis. The first symptoms of this disease appeared for the first time at the beginning of the third trimester of her second pregnancy, and diagnosis was established on the basis of typical clinical presentation, confirmed by a water deprivation test. Diabetes insipidus resulted from the increased activity of vasopressinase, caused by hemochromatosis-induced liver dysfunction, the presence of which was confirmed between the pregnancies by liver biopsy and identification of the HFE gene mutation. Subsequent desferrioxamine treatment resulted in a less severe clinical course of diabetes insipidus in the last patient's pregnancy. In both pregnancies, the patient was successfully treated with oral desmopressin, which is resistant to degradation by placental vasopressinase. Although unrecognized pituitary disorders may pose a serious health problem to the mother and fetus, hemochromatosis-induced diabetes insipidus, as the case of our patient demonstrates, if effectively diagnosed and treated, cannot be regarded as a contraindication for pregnancy.
\end{abstract}

Key words: Diabetes insipidus, Hemochromatosis, Pregnancy, Clinical picture, Diagnosis and management

DIABETES INSIPIDUS (DI) is a rare endocrine disorder, with an incidence in a general population assessed on one case per 25,000-30,000 people [1, 2]. Even rarer this syndrome occurs during pregnancy and therefore only a limited number of cases of DI during gestation have been described in literature. DI may either be a continuation of the syndrome discovered before pregnancy or, and these cases undoubtedly dominate, or it may appear for the first time in pregnancy and disappear after delivery [2-5]. There are three main types of pregnancy-induced DI. The most common of them is observed in the third trimester of pregnancy and affects women free of any earlier water balance abnormalities. It is usually found in subjects with HELLP (hemolysis, elevated liver enzymes and low platelets) syndrome

Received Apr. 26, 2010; Accepted Sep. 10, 2010 as K10E-125

Released online in J-STAGE as advance publication Oct.13, 2010

Correspondence to: Robert Krysiak, Department of Internal Medicine and Clinical Pharmacology, Medical University of Silesia, Medyków 18, 40-752 Katowice, Poland.

E-mail: r.krysiak@interia.pl or acute fatty liver of pregnancy. This type is associated with the increased activity of placental vasopressinase, the activity of which is strongly elevated (up to 300 times) due to the reduction of its hepatic degradation $[4,5]$. This type usually does not recur in the following pregnancies $[4,5]$. The second type affects women with partial defect of vasopressin secretion. These women, who do not experience any symptoms of DI before pregnancy, suddenly, particularly within the second trimester, observe a massive increase in diuresis $[2,6]$. This type results from a pregnancy-induced increase in the degradation of vasopressin, the reserves of which are insufficient $[2,6]$. Finally, the third type of pregnancy-induced DI is associated with abnormally high levels of both vasopressin and prostaglandin $E_{2}$, and probably results from inhibiting by this second agent vasopressin action at the level of its receptor $[4,6]$.

The major form of treatment of this disease is administering desmopressin. Exogenous vasopressin is not only often ineffective (fast degradation by placental 
Table 1 Baseline biochemical characteristics of the patient

\begin{tabular}{lccc}
\hline Parameter & Unit & Concentration & Normal limits \\
\hline Alanine aminotransferase & $\mathrm{U} / \mathrm{L}$ & 75.0 & below 25 \\
Aspartate aminotransferase & $\mathrm{U} / \mathrm{L}$ & 73.5 & below 25 \\
$\gamma$-glutamyl transpeptidase & $\mathrm{U} / \mathrm{L}$ & 83.1 & below 50 \\
Total bilirubin & $\mathrm{mg} / \mathrm{dL}$ & 1.7 & $0.3-1.2$ \\
Total protein & $\mathrm{g} / \mathrm{dL}$ & 52.0 & $60-80$ \\
Albumin & $\mathrm{g} / \mathrm{dL}$ & 3.1 & $3.3-5.6$ \\
Glucose & $\mathrm{mg} / \mathrm{dL}$ & 83.4 & $70-95$ \\
Creatinine & $\mu \mathrm{mol} / \mathrm{L}$ & 112 & $60-130$ \\
Urea nitrogen & $\mathrm{mg} / \mathrm{dL}$ & 35 & $7-20$ \\
Sodium & $\mathrm{mmol} / \mathrm{L}$ & 148 & $135-142$ \\
Potassium & $\mathrm{mmol} / \mathrm{L}$ & 3.5 & $3.3-5.2$ \\
\hline
\end{tabular}

vasopressinase) and shows a short half-life, but also may affect uterine contractions [7]. Desmopressin by a selective influence on $\mathrm{V}_{2}$ receptors does not increase blood pressure and uterine contractions and its half-life is about 12 hours [7].

In this paper we present the case of gestational DI induced by hemochromatosis which occurred in two of three patient's pregnancies.

\section{Case Report}

A thirty-five-year-old female referred to our ward in the 28 week of her second pregnancy from another unit for further evaluation and treatment. The only health problem the patient had suffered from before gestation was long-lasting chronic rhinitis. Her first pregnancy, six years earlier was uneventful, and ended in term by spontaneous vaginal delivery. On admittance, she was asthenic, complained of polydipsia and polyuria with pronounced nocturia. Her skin and mouth were completely dry, the thyroid gland was not palpable and there was no galactorrhea. The liver was slightly enlarged ( $2 \mathrm{~cm}$ below the costal margin). Uterine size corresponded to the period of gestation. Blood pressure was $95 / 60 \mathrm{mmHg}$ while heart rate 87 beats per minute. Laboratory data on admission were as follows: hematocrit: $47 \%$, hemoglobin: $15.8 \mathrm{~g} / \mathrm{dL}$, erythrocytes: 5,100,000/mL, platelets 250,000/mL, leukocytes: 6,200/mL. Plasma sodium was elevated (148 mmol/L) while plasma potassium was within normal limits (3.5 mmol/L). Glycemia and plasma creatinine were normal, whereas the blood urea nitrogen was elevated (Table 1). Biochemical investigations revealed mildly deranged liver function tests (slightly increased activity of alanine and aspartate aminotransferase, and $\gamma$-glutamyl transpeptidase $(\gamma \mathrm{GT}))$ Total bilirubin was slightly increased. Total protein and albumin levels were reduced (Table 1), despite clinical symptoms of dehydratation. Apart from reduced urine gravity (1.004) and urine osmolarity (84 mOsm/kg), no changes in urinalysis were found. Despite high plasma osmolarity (315 mOsm $/ \mathrm{kg}$ ), vasopressin levels were markedly reduced $(0.41 \mathrm{pg} / \mathrm{mL}$, normal limits: 0.6-4.5 $\mathrm{pg} / \mathrm{mL}$ ) (Table 2). The 24-h urinary output was $14 \mathrm{~L}$, while the oral fluid intake: $16 \mathrm{~L}$. These symptoms suggested the presence of DI and therefore, an overnight water deprivation test was performed. The test lasting 11 hours was stopped because of three consecutive sampled differed by less than $30 \mathrm{mOsm} / \mathrm{kg}$ and owing to weight loss of $3 \%$. At the end of this test urine osmolarity reached the value of $150 \mathrm{mOsm} / \mathrm{kg}$, increasing after injection of $1 \mu \mathrm{g}$ of desmopressin to $525 \mathrm{mOsm} /$ $\mathrm{kg}$. Vasopressinase activity was $875 \mathrm{U} / \mathrm{L}$ (normal limits for pregnant women: 75-280 U/L). $\mathrm{T}_{1}$-weighted MR images of the pituitary gland revealed the loss of hyperintensive signal of the posterior lobe, thickening of the pituitary stalk and unchanged the anterior lobe (Fig. 1). Gradient-echo (GRE) T2-weighted imaging failed to show the reduction of signal intensity.

Endocrine evaluation revealed no pathological changes in the activity of the growth hormone-IGF-I axis, the hypothalamic-pituitary-thyroid axis and the hypothalamic-pituitary-adrenal axis (Table 2). Plasma iron concentration and iron binding capacity were, respectively, $45 \mu \mathrm{mol} / \mathrm{L}$ (normal range: $10-40 \mu \mathrm{mol} / \mathrm{L}$ ) and $39 \mu \mathrm{mol} / \mathrm{L}$ (normal range: $45-80 \mu \mathrm{mol} / \mathrm{L}$ ). Plasma ferritin was raised $(937 \mu \mathrm{g} / \mathrm{L}$; normal range: $15-250$ $\mu \mathrm{g} / \mathrm{L})$. Although the picture suggested hemochromato- 

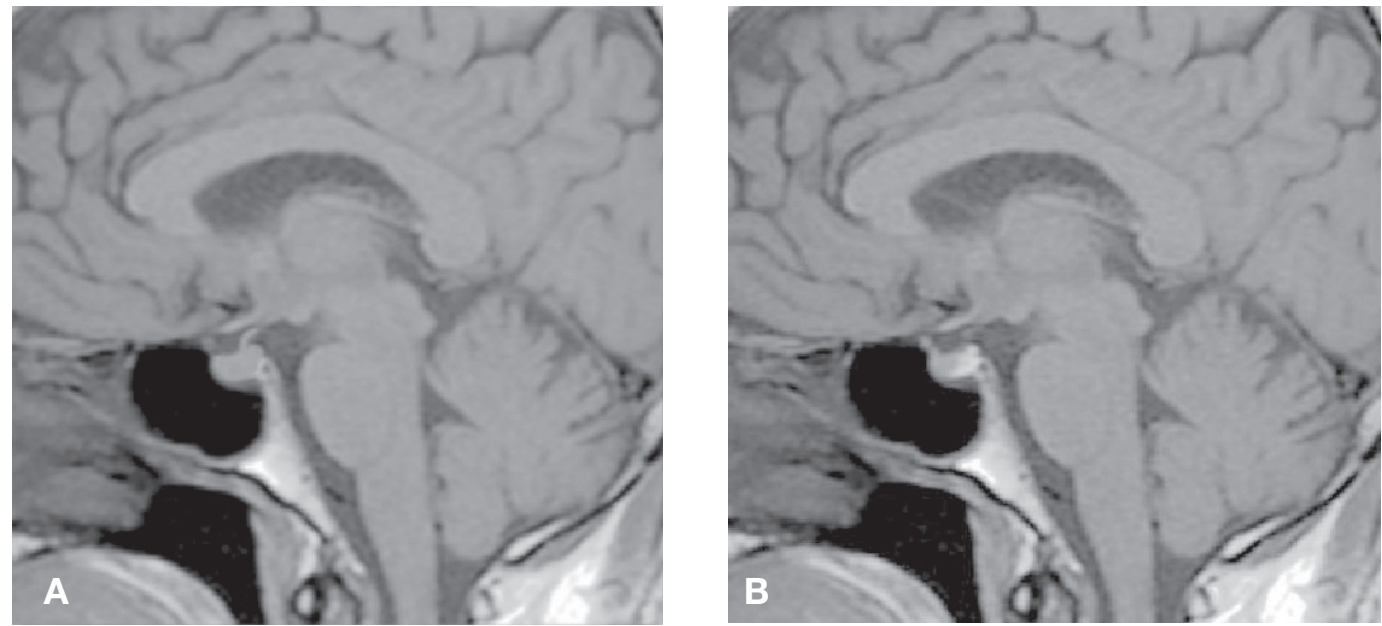

Fig. 1 (A) Sagittal precontrast T1-weighted image demonstrates the loss of posterior pituitary hyperintensity and thickening of the pituitary stalk. (B) Normal sagittal precontrast T1-weighted image after delivery and desferrioxamine treatment.

Table 2 Hormonal characteristics of the patient

\begin{tabular}{|c|c|c|c|c|c|}
\hline Hormone (Unit) & $\begin{array}{l}\text { Normal limits in } \\
\text { non-pregnant state }\end{array}$ & Second pregnancy ${ }^{1}$ & $\begin{array}{l}\text { Between the } \\
\text { pregnancies }^{2}\end{array}$ & Third pregnancy ${ }^{1}$ & $\begin{array}{l}\text { After the third } \\
\text { pregnancy }^{2}\end{array}$ \\
\hline Prolactin $(\mu \mathrm{g} / \mathrm{L})$ & $5.0-25.0$ & Not investigated ${ }^{3}$ & 21.4 & Not investigated ${ }^{3}$ & 18.6 \\
\hline $\begin{array}{l}\text { GH in the insulin } \\
\text { tolerance test }(\mu \mathrm{g} / \mathrm{L})\end{array}$ & $>5.0$ & Not investigated ${ }^{4}$ & 10.4 & Not investigated ${ }^{4}$ & 11.2 \\
\hline $\begin{array}{l}\text { GH in the oral glucose } \\
\text { tolerance test }(\mu \mathrm{g} / \mathrm{L})\end{array}$ & $<1.0$ & Not investigated ${ }^{3}$ & 0.22 & Not investigated ${ }^{3}$ & 0.18 \\
\hline IGF-I (ng/mL) & $75.0-340.0^{5}$ & 325 & 203.4 & 310 & 198.4 \\
\hline ACTH (ng/L) & $20.0-60.0$ & Not investigated ${ }^{3}$ & 25.3 & Not investigated ${ }^{3}$ & 30.1 \\
\hline TSH (mIU/L) & $0.4-4.5$ & 0.83 & 1.43 & 0.92 & 1.65 \\
\hline $\mathrm{FSH}^{6}(\mathrm{IU} / \mathrm{L})$ & $3.4-12.5$ & Not investigated $^{7}$ & 5.1 & Not investigated $^{7}$ & 5.5 \\
\hline $\mathrm{LH}^{6}(\mathrm{IU} / \mathrm{L})$ & $2.3-12.7$ & Not investigated ${ }^{7}$ & 4.6 & Not investigated ${ }^{7}$ & 5.0 \\
\hline $\begin{array}{l}\text { Plasma cortisol in the } 250-\mu \mathrm{g} \\
\text { cosyntropin test }(\mu \mathrm{g} / \mathrm{dL})\end{array}$ & $>19.6$ & Not investigated ${ }^{3}$ & 27.2 & Not investigated ${ }^{3}$ & 27.5 \\
\hline Urine free cortisol ( $\mu \mathrm{g} /$ day) & $20.0-90.0^{8}$ & 180 & 53 & 174 & 49 \\
\hline Free thyroxine (pmol/L) & $12.0-22.0$ & 12.9 & 18.4 & 14.1 & 17.9 \\
\hline Free triiodothyronine $(\mathrm{pmol} / \mathrm{L})$ & $2.8-6.0$ & 3.1 & 4.2 & 3.2 & 4.5 \\
\hline Vasopressin (pg/mL) & $0.6-4.5$ & 0.41 & 2.1 & 0.55 & 2.4 \\
\hline
\end{tabular}

${ }^{1}$ measurements made in the third trimester; ${ }^{2}$ measurements made after weaning; ${ }^{3}$ Pregnancy is associated with marked increase in its levels.; ${ }^{4}$ test contraindicated in pregnancy; ${ }^{5}$ the upper normal limit in the third trimester up to $500 \mathrm{ng} / \mathrm{mL} ;{ }^{6}$ early follicular phase; ${ }^{7}$ Pregnancy is associated with marked reduction in its levels.; ${ }^{8}$ in the late pregnancy the upper normal limit 2-3 times higher than in non-pregnant state 


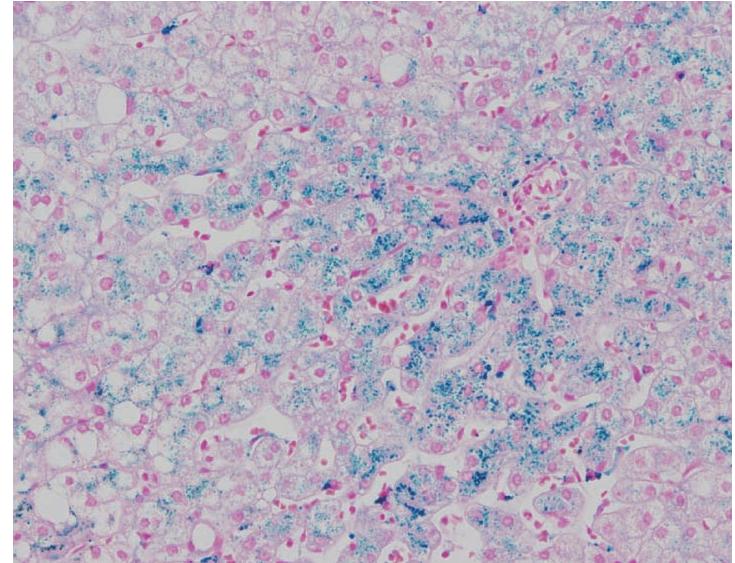

Fig. 2 Core needle biopsy of the liver. Perl's Prussian Blue staining revealed massive hepatic iron accumulation.

sis, the patient, anxious regarding the exposure of the fetus on diagnostic procedures, decided to postpone the further diagnosis on the time after delivery. After establishing the diagnosis of DI the patient was given oral tablets of desmopressine acetate (Minirin) administered at starting doses of $200 \mu \mathrm{g}$ at bedtime (to prevent nocturia), which had to be titrated up to $300 \mu \mathrm{g}$ three times a day. Her sodium plasma levels started to decrease reaching the value of $140 \mathrm{mmol} / \mathrm{L}$ after 15 days, and this was accompanied by a normalization in urine volume. There was no uterotonic or other reactions to desmopressin, and enhanced thirst disappeared within several days. A week before the calculated term, she gave spontaneous birth to a fully developed baby. Two weeks after delivery symptoms of vasopressin deficiency disappeared, plasma vasopressin levels normalized (Table 2) and plasma vasopressinase activity decreased by more than 450 times (to 1.9 $\mathrm{U} / \mathrm{L}$ ) and therefore demopressin treatment was withdrawn. Liver biopsy, performed a month after delivery, revealed hemosiderosis of hepatic cells stained with Perls Prussian blue (Fig. 2). The calculated hepatic iron index was $3.02 \mu \mathrm{mol} / \mathrm{g} /$ year (normal limits below 2.0). Final diagnosis of hemochromatosis was supported by finding the typical C282Y mutation of the HFE gene (Fig. 3). The patients was prescribed with desferrioxamine (2 g daily subcutaneously) and a diet poor in iron was advised, which resulted in an improvement of patient's condition and normalization of the results of biochemical tests (including liver enzyme activities), and the treatment was continued for the following four years, until her next pregnancy. After pregnancy was

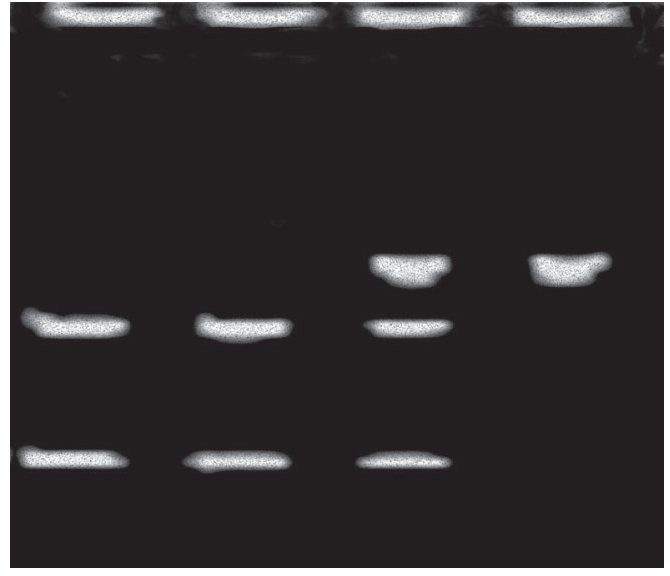

Fig. 3 Genotype analysis. In the presence of the C282Y mutation, SnaBI cleaves the 486-bp PCR product into 320-bp and 166-bp fragments. From the left side, lane A (both the 320-bp and the 166-bp fragment are visible) - the patient, lane B (both the 320-bp and the 166-bp fragments are visible) - C282Y homozygote control, lane C (486-bp, 320-bp and 166-bp fragments are visible) C282Y heterozygote control, lane D (only the 486-bp fragment is visible) - normal control.

diagnosed, the consulting gastroenterologist stopped further administration of desferrioxamine, replacing it with phlebotomies, conducted twice weekly, together with infusions of crystalloids and colloids to replace lost fluids. The patient appeared at our department about the 24 week of her third pregnancy, when symptoms of overt DI, having, however, less intensity as in previous pregnancy (diuresis and fluid intake about 8-10 L). Mildly reduced levels of plasma vasopres$\sin (0.55 \mathrm{pg} / \mathrm{mL})$ were accompanied by vasopressinase activity moderately exceeding the upper normal limit (385 U/L). Therefore, treatment with oral desmopressin was again introduced but this time smaller doses of desmopressin ( $400 \mu \mathrm{g}$ daily) were enough to avoid excessive diuresis and dehydratation, and to effectively control DI. In the 38th week she gave birth to another healthy infant. During several days after delivery the symptoms of DI disappeared, which enabled us to stop further treatment with desmopressin. Clinical improvement was paralelled by a normalization of plasma vasopressin content and a decrease in vasopressinase activity to the values observed in the non-pregnant state (1.1 $\mathrm{U} / \mathrm{L}$ ). Control MR of the pituitary revealed the presence of posterior pituitary bright spot.

Presently, 2 years after the last delivery, the patient feels well and does not experience any symptoms of DI. 
She receives $2 \mathrm{~g}$ of desferrioxamine daily in slow subcunaeous infusion by means of a portable infusion pump. On desferrioxamine treatment, which is well tolerated, liver biochemistry and ultrasonography are normal.

\section{Discussion}

This report describes for the first time hemochromatosis patient who developed gestational DI during two following pregnancies.

Although it cannot be completely excluded that DI simply coincided with hemochromatosis, this situation is rather unlikely. Firstly, taking into account the frequency of DI $(1: 30,000)[2,3]$ and hemochromatosis $(1: 300)[8,9]$ in the American population the probability of their coincidence is assessed on 1 case per $9,000,000$. In fact, this probability is even much lower, as more than 90 percent of all individuals suffering from hemochromatosis are males, while the frequency of hemochromatosis in Poland is less than in the United States. Secondly, no transient DI was observed in the patient's first pregnancy, when she did not experience any symptoms of hemochromatosis. Thirdly, the intensity of DI in the second pregnancy was higher than during the third one, probably because during the former she was unaware of hemochromatosis, while before and during the third gestation hemochromatosis was effectively treated with desferrioxamine and phlebotomies.

Interestingly, the presence of hemochromatosis is associated with various endocrine abnormalities, including diabetes mellitus, adrenal insufficiency, hypothyroidism, hypoparathyroidism, DI, hypogonadotropic hypogonadism and testicular atrophy [10]. There are some pieces of evidence that iron deposits may concentrate in the anterior lobe of the pituitary [11, 12], disturbing hypophyseal hormone synthesis and release [10]. In our patient we did not observe any disturbances in the release of TSH, ACTH, gonadotropins, GH and prolactin, when assessed after pregnancy and lactation. Although it was not the case in our subject, it is possible that secondary adrenal insufficiency may mask the symptoms of DI, explaining poverty of data on the occurrence of DI in hemochromatosis.

To the best of our knowledge our patient is the first one with hemochromatosis who developed vassopressin-dependent DI. In literature, there is only one report of nephrogenic DI in a male patient with hemochromatosis. In the study by Okumura et al. [13], the authors observed persistent polyuria despite accurate basal secretion of vasopressin. The fact that urinary concentrating capacity was not corrected by supraphysiologic doses of exogenous desmopressin suggests a nephrogenic form of DI in this subject, supported by the presence of iron deposits in the tubular epithelium of distal urinary tubules and collecting ducts. Similar model of nephrogenic DI was experimentally induced in animals [14]. Therefore, we cannot exclude that resistance to vasopressin contributed to the pathogenesis of DI in our subject. However, the fact that desmopressin treatment restored normal diurnal urine loss indicates that the involvement of the renal resistance, if at all present, was very small.

Although at first glance hemochromatosis-induced DI might result from the presence of iron deposits in the pituitary and stalk, this does not seem to be the case in our patient. We assume that DI in this subject resulted from enhanced breakdown of endogenous vasopressin resulting from disturbed liver degradation of vasopressinase. This explanation is supported by both laboratory and image findings. Vasopressinase activity was much higher in the discussed patient than observed in normal pregnancy. The anterior lobe of the pituitary gland was free from iron deposits on Gradient-echo (GRE) T2-weighted imaging, shown to be the most sensitive sequence for detecting excess pituitary iron deposition [15]. Moreover, hyperintense signal on T1-weighed MR images, absent because of markedly decreased vasopressin content, restored after delivery, suggesting at least a partial increase in vasopressin synthesis.

Desmopressin is resistant to ingestion by placental vasopressinase and therefore this agent seem to be more effective than unmodified vasopressin and therefore seem to be the treatment option of choice in pregnant women [7]. In most of so far described cases, subjects were treated with intranasal application of this agent, which seems to be safe, as no serious adverse effects on pregnancy, delivery and postpartum period and on the health's condition of both the mother and fetus were observed. In our patient we had to administered desmopressin by an oral route, because of chronic rhinitis, which disturbs its pharmacokinetics. Its safety and efficacy during two subsequent pregnancies clearly indicates that oral desmopressin may be considered an alternative to intranasal route of administration.

Despite DI in the second pregnancy, the patient conceived for the third time. What is more, DI in the subsequent pregnancy had a milder course, probably 
because after establishing the correct diagnosis our patient was effectively treated. This finding as well as a small number of data on the recurrence of DI in subsequent pregnancies indicate that the presence of this disorder during gestation does not preclude patients from future ones.

To sum up, our report presents the case of DI in a woman with unrecognized and consequently untreated hemochromatosis, which ended in a successful deliv- ery after treatment with oral desmopressin. This indicates that hemochromatosis complicated by DI, if effectively diagnosed and treated, cannot be regarded as a contraindication for pregnancy.

\section{Conflict of Interest}

The authors declare no conflict of interest.

\section{References}

1. Robertson GL (1995) Diabetes insipidus. Endocrinol Metab Clin North Am 24: 549-572.

2. Ananthakrishnan S (2009) Diabetes insipidus in pregnancy: etiology, evaluation, and management Endocr Pract 15: 377-382.

3. Foyouzi N, Frisbaek Y, Norwitz ER (2004) Pituitary gland and pregnancy. Obstet Gynecol Clin North Am 31: 873-892.

4. Sainz Bueno JA, Villarejo Ortíz P, Hidalgo Amat J, Caballero Fernández V, Caballero Manzano M, Garrido Teruel R (2005) Transient diabetes insipidus during pregnancy: a clinical case and a review of the syndrome. Eur J Obstet Gynecol Reprod Biol 118: 251-254.

5. Kalelioglu I, Kubat Uzum A, Yildirim A, Ozkan T, Gungor F, Has R (2007) Transient gestational diabetes insipidus diagnosed in successive pregnancies: review of pathophysiology, diagnosis, treatment, and management of delivery. Pituitary 10: 87-93.

6. Lindheimer MD (2005) Polyuria and pregnancy: its cause, its danger. Obstet Gynecol 105 (5 Pt 2): 11711172.

7. Ray JG (1998) DDAVP use during pregnancy: an analysis of its safety for mother and child. Obstet Gynecol Surv 53: 450-455.

8. Janssen MC, Swinkels DW (2009) Hereditary haemo- chromatosis. Best Pract Res Clin Gastroenterol 23: 171-183.

9. Fix OK, Kowdley KV (2008) Hereditary hemochromatosis. Minerva Med 99: 605-617.

10. Pietrangelo A (2007) Hemochromatosis: an endocrine liver disease. Hepatology 46: 1291-1301.

11. Bergeron C, Kovacs K (1978) Pituitary siderosis: a histologic, immunocytologic and ultrastructural study. Am J Pathol 93: 295-310.

12. Wahid S, Ball S (2001) The pituitary gland and hereditary haemochromatosis. Lancet 357: 115.

13. Okumura A, Kondo K, Hirai C, Nishimura H, Tamai H, Kawarazaki F, Ichikawa M, Mizuno M, Oiso Y, Yamamoto M (2002) Nephrogenic diabetes insipidus associated with hemochromatosis. Am J Kidney Dis 40: 403-406.

14. Zhou XJ, Vaziri ND, Pandian D, Wang ZQ, Mazowiecki M, Liao SY, Oveisi F (1996) Urinary concentrating defect in experimental hemochromatosis. J Am Soc Nephrol 7: 128-134.

15. Sparacia G, Iaia A, Banco A, D'Angelo P, Lagalla R (2000) Transfusional hemochromatosis: quantitative relation of MR imaging pituitary signal intensity reduction to hypogonadotropic hypogonadism. Radiology 215: 818-823. 